\title{
Dynamics of the Parkinsonian Striatal Microcircuit: Entrainment into a Dominant Network State
}

\author{
Omar Jáidar, Luis Carrillo-Reid, Adán Hernández, René Drucker-Colín, José Bargas, and Arturo Hernández-Cruz \\ Instituto de Fisiología Celular, División de Neurociencias, Universidad Nacional Autónoma de México, México City, DF CP 04510 México
}

Neuronal synchronization in basal ganglia circuits plays a key role in the encoding of movement, procedural memory storage and habit formation. Striatal dopamine (DA) depletion during Parkinsonism causes abnormal synchronization in corticobasal ganglia loops resulting in motor dysfunction. However, the dynamics of the striatal microcircuit underlying abnormal synchronization in Parkinsonism is poorly understood. Here we used targeted whole-cell recordings, calcium imaging allowing the recording from dozens of cells simultaneously and analytical approaches, to describe the striking alterations in network dynamics that the striatal microcircuit undergoes following DA depletion in a rat model of Parkinson disease (PD): In addition to a significant enhancement of basal neuronal activity frequent periods of spontaneous synchronization were observed. Multidimensional reduction techniques of vectorized network dynamics revealed that increased synchronization resulted from a dominant network state that absorbed most spontaneously active cells. Abnormal synchronous activity can be virtually abolished by glutamatergic antagonists, while blockade of GABAergic transmission facilitates the engagement of striatal cell assemblies in the dominant state. Finally, a dopaminergic receptor agonist was capable of uncoupling neurons from the dominant state. Abnormal synchronization and "locking" into a dominant state may represent the basic neuronal mechanism that underlies movement disorders at the microcircuit level.

\section{Introduction}

The synchronous firing of neurons has been proposed as a mechanism to generate representations of perceptual objects, cognitive functions and motor programs (Singer, 1999; Uhlhaas et al., 2009). Correlated activity of different neurons between the cortex and the basal ganglia (BG) follows reentrant loops whose cyclical activity encode movement, procedural memories, and habit formation (Barnes et al., 2005; DeLong and Wichmann, 2007; Fries et al., 2007; Carrillo-Reid et al., 2008; Graybiel, 2008). Spatiotemporal patterns of correlated activity can be induced in the striatal microcircuit and shown to be dependent upon both synaptic and intrinsic neuronal properties (Carrillo-Reid et al., 2008, 2009a,b).

In pathological states such as Parkinson disease, BG neurons change their behavior (Tseng et al., 2001) exhibiting enhanced and abnormal synchronization (Costa et al., 2006; Fuentes et al., 2009; Walters and Bergstrom, 2009). Dopamine (DA) depletion changes global functions, promoting anomalous consequences

Received March 17, 2010; revised June 3, 2010; accepted July 5, 2010.

This work was supported by a program project Grant IMPULSA 03 [Universidad Nacional Autónoma de México (UNAM)] to J.B., E.G., A.H.-C., and R.D.-C., Grants 98004 to E.G. and 79763 to A.H.-C. [Consejo Nacional de Ciencia y Tecnologia (CONACyT)-Mexico), and Grants IN-205610 to J.B., IN-206010 to E.G., and IN227307 to A.H.-C. [Dirección General de Asuntos del Personal Académico (DGAPA)-UNAM]. 0.J. and L.C.-R. received Ph.D. fellowships from CONACyT-México. A.H. received a postdoctoral fellowship from CONACyT. 0.J. participated in this work in partial fulfillment of the requirements for the Ph.D. degree in Biomedical Sciences at the Universidad Nacional Autónoma de México. We thank Antonio Laville, Dagoberto Tapia, and Nicolás Jiménez for technical support, and Jean-Pascal Morin for helpful comments on the manuscript. We are indebted to Claudia Rivera, Head of the Animal Facility, for continuous advice, and Ana María Escalante for expert computing support.

Correspondence should be addressed to José Bargas, Instituto de Fisiología Celular, División de Neurociencias, Universidad Nacional Autónoma de México, P.0. Box 70-253, México City, DF 04510 México. E-mail: jbargas@ if.unam.mx.

DOI:10.1523/JNEUROSCI.1380-10.2010

Copyright $\odot 2010$ the authors $\quad 0270-6474 / 10 / 3011326-11 \$ 15.00 / 0$
(Magill et al., 2001; Ni et al., 2001; Wilson et al., 2006): bradykinesia, akinesia, tremor and muscular rigidity are attributed to abnormal synchronization (Brown, 2007; Hammond et al., 2007; Fuentes et al., 2009; Walters and Bergstrom, 2009; Zold et al., 2009). Nevertheless, a study of the changes of neuronal activity, explaining abnormal synchronization in the striatal microcircuit or, any other BG local network under DA depletion, is missing. Such correlation would support the hypothesis that global circuits are dynamically built by the coordinated actions of several similar interconnected microcircuits (Uhlhaas et al., 2009) that integrate their activity.

It has been shown previously, in vitro and in vivo, that spontaneous firing and synaptic activity of striatal neurons is enhanced with respect to the control condition after DA depletion (Galarraga et al., 1987; Tang et al., 2001; Tseng et al., 2001; Liang et al., 2008). Enhanced activity is characterized by recurrent bursting accompanied by numerous periods of spontaneous synchronization not seen in the controls (Zold et al., 2009). However, microcircuit dynamics in these conditions has not been described. Previously, we reported that microcircuit dynamics can be induced in control non-DAdepleted corticostriatal slices during NMDA administration. NMDA-induced microcircuit dynamics consists in spatiotemporal patterned and correlated activity that travels among diverse network states (Carrillo-Reid et al., 2008, 2009a). Here we use whole-cell recordings, calcium imaging techniques and analytical approaches to first compare control and unilateral DA-depleted preparations, and second, to discuss the differences between spontaneous circuit dynamics found during DA-depletion and induced dynamics during NMDA from our preceding work.

Dimensional reduction of vectorized network activity revealed that episodes of synchronous activity in DA-depleted slices 
result from a dominant network state that absorbs most active cells. Blockade of glutamatergic transmission reduced hyperactivity and abnormal synchronization of the pathological network, while blockade of GABAergic transmission facilitated the entrainment of the circuit into the anomalous dynamics. In addition, a dopamine receptor agonist was capable of disengaging the neurons from the dominant state, partially reestablishing dynamics between diverse network states. We demonstrate a remarkable functional reconfiguration of the striatal microcircuit after DA-depletion. This pathological configuration could be partially reverted after dopamine agonist administration making our preparation a suitable approach to test the effect of different pharmacological agents to reduce the signs of movement disorders.

\section{Materials and Methods}

Rat model of Parkinson disease. Anesthetized Wistar male rats (postnatal day 12-25) were injected with $2 \mu \mathrm{l}$ of 6-hydroxydopamine (6-OHDA) (Sigma; $4 \mu \mathrm{g} / \mu \mathrm{l}$ in $0.9 \% \mathrm{NaCl}, 0.5 \% \mathrm{C}_{6} \mathrm{H}_{8} \mathrm{O}_{6}$ ) into the right substantia nigra (SN) at $1 \mu \mathrm{l} / \mathrm{min}$ at stereotaxic coordinates: anteroposterior, 3.9; lateral, 1.8; ventral, $6.7 \mathrm{~mm}$ (Dunnett et al., 1981). After 1 week postinjection, rats were treated with $4 \mathrm{mg} / \mathrm{kg}$ i.p. amphetamine and ipsilateral turns were counted for $90 \mathrm{~min}$ with an automatic apparatus. Animals showing $>500$ ipsilateral turns were used for the experiments, which were performed 11-15 d after the lesion. This rotational score corresponds to $>97 \%$ lesion of SN dopaminergic cells (Dunnett et al., 1981; Ingham et al., 1998; Grant and Clarke, 2002).

Tyrosine hydroxylase immunoreactivity. The degree of lesion was verified using tyrosine hydroxylase $(\mathrm{TH})$ immunostaining in a subset of rats. One week after 6-OHDA injection, rats were perfused transcardially with $4 \%$ paraformaldehyde in $0.1 \mathrm{~m}$ phosphate buffer, $\mathrm{pH} 7.3$. Then the brain was removed and coronal slices $40 \mu \mathrm{m}$ in thickness were obtained with a cryostat (Leica CM 1900). Slices were incubated overnight with rabbit anti-TH primary antibody (Millipore Bioscience Research Reagents; AB152, 1:1000) in PBS containing 10\% normal donkey serum (NDS) and $0.3 \%$ Triton X-100 (Tx) at $4^{\circ} \mathrm{C}$. After being washed in PBS, the sections were incubated for $2 \mathrm{~h}$ at room temperature with biotinylated donkey anti-rabbit IgGs diluted 1:200 in PBS-Tx containing 1\% NDS. The sections were then cleansed and reacted with the avidin-biotin peroxidase complex at room temperature for $2 \mathrm{~h}$. Bound peroxidase enzyme activity

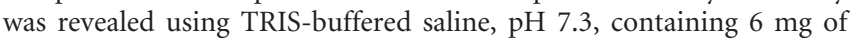
3,3-diaminobenzidine tetrahydrochloride $(\mathrm{DAB})$ and $0.1 \mathrm{ml}$ of $3 \%$ hydrogen peroxide in $10 \mathrm{ml}$ of PBS. Phase contrasted images were captured with a digital camera mounted on an upright microscope.

Preparation of corticostriatal slices. Transverse corticostriatal slices (200-250 $\mu \mathrm{m}$ in thickness), were obtained either from control or 6-OHDA-treated rats (postnatal day 23-29) as previously described (Kawaguchi et al., 1989; Vergara et al., 2003). All procedures conformed to the guidelines of the Universidad Nacional Autónoma de México's Animals Scientific Procedures Committee. Animals were anesthetized via intraperitoneal sodium pentobarbital $(1 \mathrm{ml} / 2.5 \mathrm{~kg})$ and perfused transcardially using ice-cold $\left(4^{\circ} \mathrm{C}\right)$ saline solution containing the following (in mM): 124 choline chloride, $2.5 \mathrm{KCl}, 1.3 \mathrm{MgCl}_{2}, 26 \mathrm{NaHCO}_{3}, 1.2$ $\mathrm{NaH}_{2} \mathrm{PO}_{4}, 2.4 \mathrm{CaCl}_{2}$, and 10 glucose. Solution was saturated with $95 \%$ $\mathrm{O}_{2}$ and $5 \% \mathrm{CO}_{2} ; \mathrm{pH}=7.4 ; 298 \mathrm{mOsm} / \mathrm{L}$. Then slices were cut in ice-cold saline $\left(4^{\circ} \mathrm{C}\right)$ containing the following (in $\mathrm{mm}$ ): $123 \mathrm{NaCl}, 3.5 \mathrm{KCl}, 1$ $\mathrm{MgCl}_{2}, 1 \mathrm{CaCl}_{2}, 26 \mathrm{NaHCO}_{3}$, and 11 glucose $\left(25^{\circ} \mathrm{C}\right.$; saturated with $95 \%$ $\mathrm{O}_{2}$ and $5 \% \mathrm{CO}_{2} ; \mathrm{pH}=7.4 ; 298 \mathrm{mOsm} / \mathrm{L}$ ). Slices were then transferred to saline where they remained for at least $1 \mathrm{~h}$ before recording at room temperature $\left(21-25^{\circ} \mathrm{C}\right)$.

Calcium imaging. Slices were incubated at $35^{\circ} \mathrm{C}$ in the dark for $20-30$ min in the presence of 10-20 $\mu \mathrm{M}$ fluo 4-AM (Invitrogen Life Sciences) and $0.1 \%$ DMSO equilibrated with $95 \% \mathrm{O}_{2}$ and $5 \% \mathrm{CO}_{2}$. Once loaded with fluo-4, slices were transferred to a perfusion chamber and perfused continuously with control saline (see above) on the stage of an upright microscope (Nikon Eclipse $80 \mathrm{i}$ ) equipped with a $10 \times$ water-immersion objective (Nikon $0.3 \mathrm{NA} ; 2 \mathrm{~mm}$ WD). Fluo- 4 was excited at $488 \mathrm{~nm}$ with monochromatic light (Polychrome V, Illumination System, TILL Photonics), and emitted fluorescence was band-passed with a Nikon B-2E/C filter set. Fluorescence images were acquired with a cooled digital CCD camera (Imago QE, TILL Photonics) under protocols written in TILL vision software 4.0 . The field of view was $800 \times 600 \mu \mathrm{m}$ in size. Short movies (100-250 s, 50-100 ms exposure and 250-500 ms/frame) were taken at time intervals of 5-20 min during one to $2 \mathrm{~h}$. Experiments were performed at room temperature. The number of fluo 4 loaded neurons in the field was determined at the end of the experiment by the application of a $5 \mathrm{~s}$ puff of a solution containing $50 \mathrm{~mm} \mathrm{KCl}, 120 \mathrm{~mm} \mathrm{NaCl}, 10 \mathrm{~mm}$ HEPES-Na and $2 \mathrm{~mm} \mathrm{CaCl}_{2}$ pH 7.4 (see Fig. $1 D$ ). This maneuver disclosed the population of excitable fluo-4-labeled neurons in the field of vision (either active or silent during the experiment). Cells active during the experiment were analyzed and the ratio of active/silent cells was obtained. Spontaneous calcium transients, together with voltage responses were recorded in some cells (see below).

Drugs. Stock solutions were prepared before each experiment and added to the perfusion solution in the final concentration indicated. $(2 R)$-amino-5-phosphonovaleric acid (APV), 6-cyano-7-nitroquinoxaline2,3-dione (CNQX), SKF 81297 and bicuculline methiodide or hydrochloride were obtained from Sigma.

Electrophysiology. Calcium imaging and simultaneous electrophysiological recordings were obtained from areas of the dorsal striatum previously shown as receiving numerous cortical fibers (Flores-Barrera et al., 2009). The firing of more than one action potential is needed to produce significant calcium transients (Fig. 1). Electrophysiological recordings were made from medium spiny neurons visualized using infrared differential interference video microscopy and a $40 \times$ water-immersion objective (COHU 4915-2000 and Eclipse FN1, Nikon Corporation). Micropipettes for whole-cell recordings were pulled (Sutter Instrument) from borosilicate glass tubes $(1.5 \mathrm{~mm}$ outer diameter, WPI $)$ and had a final resistance of 5-8 $\mathrm{M} \Omega$ filled with (mM): $112 \mathrm{KSO}_{3} \mathrm{CH}_{3}, 14 \mathrm{KCl}, 10$ HEPES, 1.1 K $\mathrm{K}_{2}$-EGTA, 1.1 ATP-Mg and 1.1 GTP-Na (pH 7.3 adjusted with $\mathrm{KOH}$; osmolarity, 287-290 $\mathrm{mOsm} \mathrm{L}^{-1}$ ). In some experiments fluo-4 salt $(20-30 \mu \mathrm{M})$ was added to the recording pipettes.

Image analysis. Image analysis and processing was performed as previously reported (Carrillo-Reid et al., 2008, 2009a), using ImageJ (v.1.4 g, National Institutes of Health), Multicell 2.0 (kindly supplied by Robert Froemke, Max-Delbrück Center for Molecular Medicine and NeuroCure Neuroscience Research Center, Berlin, Germany), MATLAB (The MathWorks, Natick, MA), and custom made programs written in IDL (Schwartz et al., 1998; Mao et al., 2001; Cossart et al., 2003).

All fluo-4 loaded neurons in a field of view were semiautomatically identified, their contours defined as regions of interest and their mean fluorescence was measured as a function of time. The changes of fluo- 4 fluorescence $\left(\mathrm{Ca}^{2+}\right.$ signals) were computed as $\left(F_{\mathrm{i}}-F_{\mathrm{o}}\right) / F_{\mathrm{o}}$, where $F_{\mathrm{i}}$ is fluorescence intensity at any frame, and $F_{\mathrm{o}}$ is resting fluorescence, i.e., average fluorescence of the first 4 frames of the movie. Calcium signals elicited by action potentials were detected based on a threshold value given by their first derivative over time ( 2.5 times the SD of the noise value). Records were inspected manually to remove from the analysis artifacts and slow calcium transients which are likely to correspond to glial cells (Ikegaya et al., 2004; Sasaki et al., 2007; Carrillo-Reid et al., 2008, 2009a).

Statistical methods. All statistical methods used have been previously described (Carrillo-Reid et al., 2008, 2009a). Briefly, to determine whether calcium transients recorded from different cells in the field were correlated, we added the number of simultaneous activations per trial. To determine whether the $p$ value of simultaneous $\mathrm{Ca}^{2+}$ transients occurred randomly, the distribution under the null hypothesis of independent transients using Monte Carlo simulations with 1000 replications were computed (Mao et al., 2001). The degree of correlation between active cells was calculated with the Jaccard correlation coefficient and crosscorrelation maps of Jaccard correlation coefficients were constructed to show the spatial coordinates and the magnitude of the correlations between all cells pairs (Fig. 2D). We also constructed pseudo-colored crosscorrelation maps to visualize the degree of correlation between cell pairs. Then we identified the sets of cells activated simultaneously over time. Monte Carlo simulations were also used to estimate the significance of 
A

\section{6-OHDA lesion}

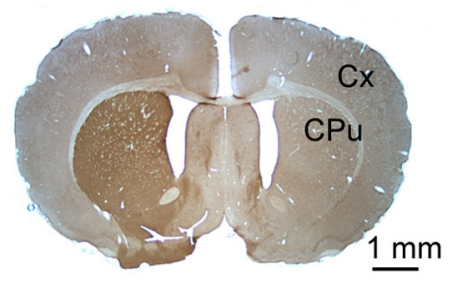

D

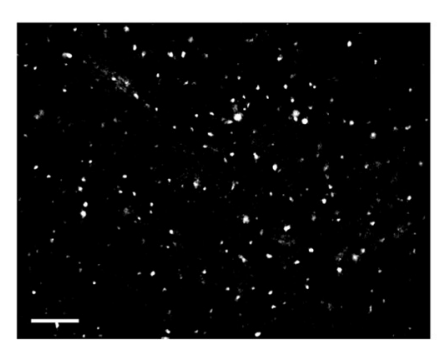

G

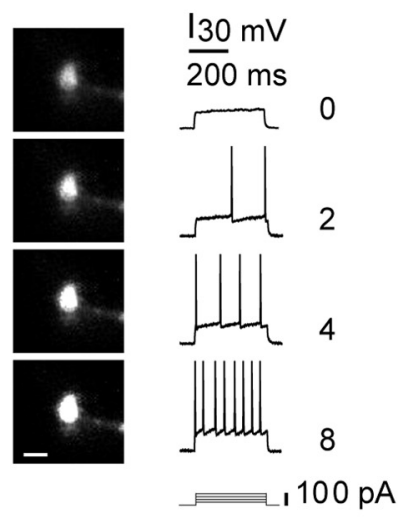

B

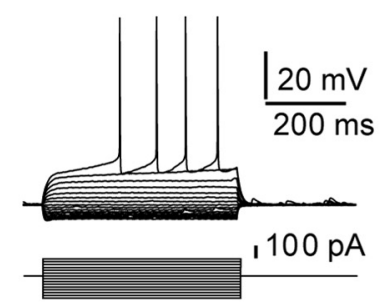

$\mathrm{E}$

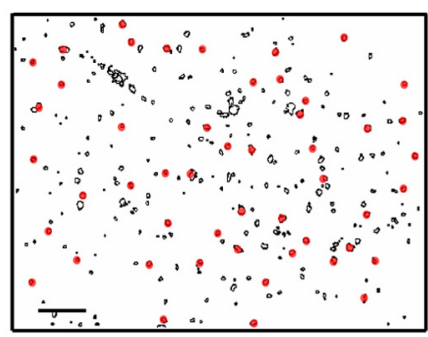

C

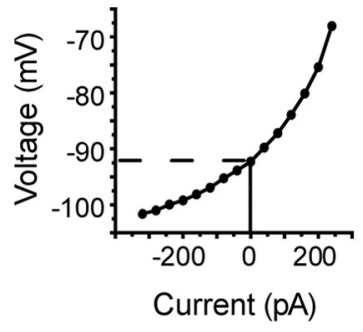

$\mathrm{F}$

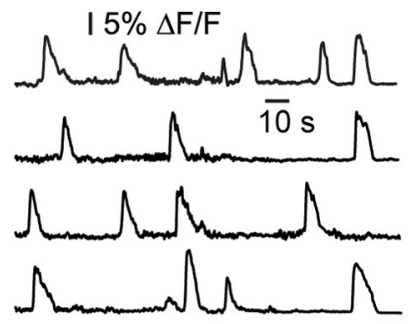

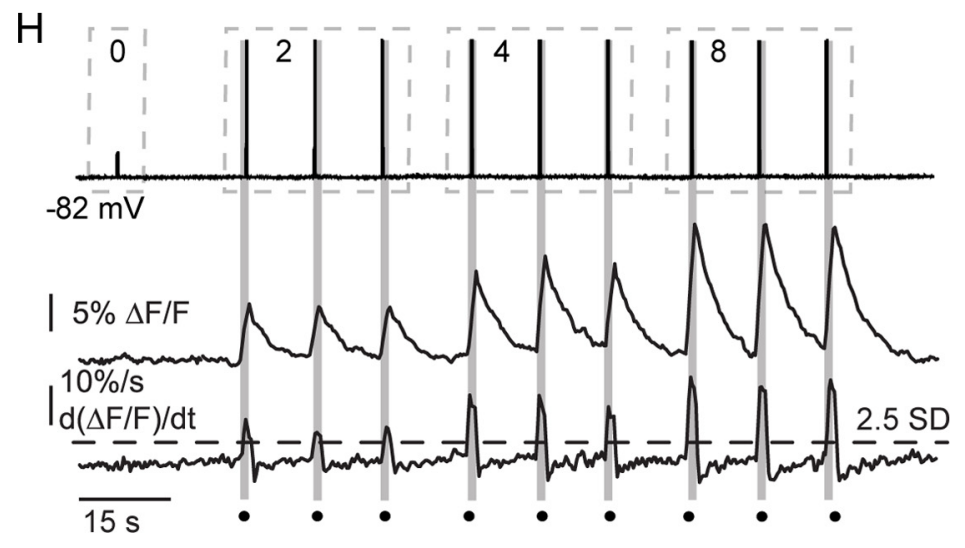

Figure 1. Optical and electrophysiological recordings from striatal neurons. $\boldsymbol{A}$, Bright-field image of a TH-immunostained coronal brain slice (brown color indicates strong reaction) from an animal that received unilaterally an injection of 6-OHDA in the substantia nigra pars compacta (see Materials and Methods). $\boldsymbol{B}$, Voltage responses (top) of a medium spiny neuron to the injection of current steps of increasing amplitude (bottom). Current-clamp recordings were obtained from a neuron in the corticostriatal slice of an untreated rat. Inward rectification and long latency to the first spike are characteristics of these neurons. C, Steady-state current-voltage relationship obtained from data in B. D. Fluorescence image from a corticostriatal slice loaded with fluo-4 AM (average of 100 frames with background subtraction, scale bar: $100 \mu \mathrm{m}$ ). $\boldsymbol{E}$, Map of 270 cells in the field shown in $\boldsymbol{D}$ that responded with a fluorescence increase after high $\mathrm{K}^{+}$depolarization. Red circles indicate the position of neurons exhibiting spontaneous calcium fluctuations (62 of 270: 23\%). F, Spontaneous calcium transients recorded from 4 such neurons. G, A fluo-4-loaded neuron targeted for electrophysiological recording was patch-clamped and current steps of increasing amplitude were applied while membrane voltage and fluorescence images were recorded. Cell was further loaded with fluo-4 pentapotassium salt through the recording patch pipette. (ell fluorescence increases proportionally to the number of action potentials (indicated) generated by membrane depolarizations of different amplitudes. Scale bar: $10 \mu \mathrm{m}$. $\boldsymbol{H}$, Simultaneous recordings of membrane depolarization, action potential generation (upper trace), calcium signaling (middle record) and first derivative of the calcium transients (bottom trace). Dashed line indicates 2.5 times the SD of the noise. Dots at the bottom indicate events where $d(\Delta F / F) / d t>2.5$ times SD. Similar data were used to build raster plots as those shown in Figure $2 \mathrm{~A}$. Calcium transients elicited by trains of two or more action potentials were considered for analysis.

their firing together. The threshold corresponded to a significance level of $p<0.01$. The peaks that were significant at $p<0.01$ were selected for further analysis.

To analyze the cell assemblies of the striatal microcircuit, $\mathrm{D} \times \mathrm{N}$ matrices were constructed, where D represents the number of active neurons in a set of experiments, and $\mathrm{N}$ denotes the firing of cells during $250 \mathrm{~ms}$ to $1 \mathrm{~s}$ time bins (spontaneous up-states last between 0.5 and $5 \mathrm{~s}$ ) (Fig. $2 \mathrm{~A}$ ). Then, peaks of synchronous activity were vectorized so that bursting activity of synchronized cells over time was associated with different vector elements. In this way each vector element was formed by the sum of differentiated calcium transients displayed by a single neuron during the time bin. We have previously shown that the first time derivative of the calcium transient corresponds to the duration of the electrophysiologically recorded burst or up-state in single neurons (Carrillo-Reid et al., 2008, 2009a). Therefore, the set of vectors denote network activity as a function of time (Brown and Williams, 2005; Sasaki et al., 2007; Carrillo-Reid et al., 2008).
To see whether two vectors have the same components, we measured the similarity index between network vectors using the normalized scalar product of all possible vector pairs, which is equivalent to the cosine of the angle between the vectors (Schreiber et al., 2003; Sasaki et al., 2006). Then, we generated matrices which denote the similarity indexes of all paired vectors and displayed them in pseudo-color scale (Figs. $3 A$ and $4 A)$. Functional states sustained by significantly correlated or synchronized bursting neuronal pools, appear as clustered structures in these plots (Sasaki et al., 2006).

The dimensionality reduction of network vectors was performed with the locally linear embedding (LLE) algorithm (Figs. 3B, 4B). LLE is an unsupervised learning technique that discloses nonlinear structures from multidimensional data (Roweis and Saul, 2000; Stopfer et al., 2003; Brown and Williams, 2005). The trajectories of our high dimensional data were not well described by linear dimensionality reduction methods (Brown and Williams, 2005; Carrillo-Reid et al., 2008, 2009a). After LLE, vectors representing the network states were projected into a space made by the first two dimensions of vectors where data points represent vectors 
A

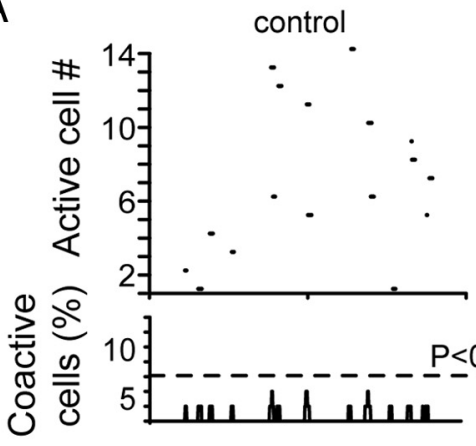

C

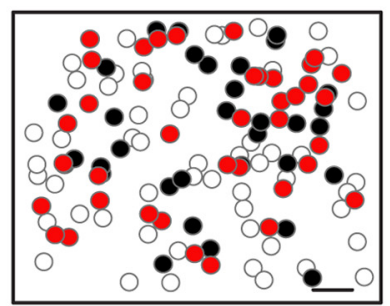

DA depleted

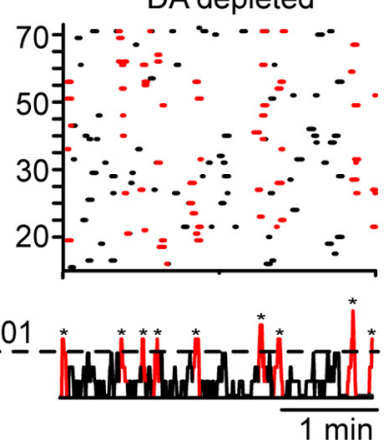

D

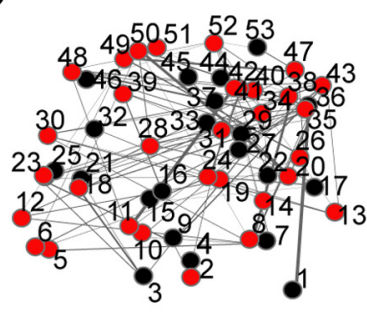

B

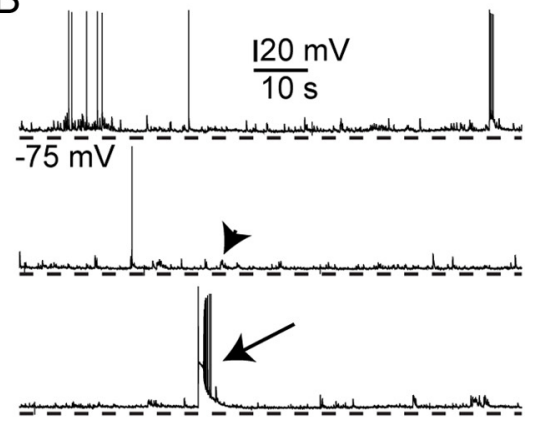

$\mathrm{E}$

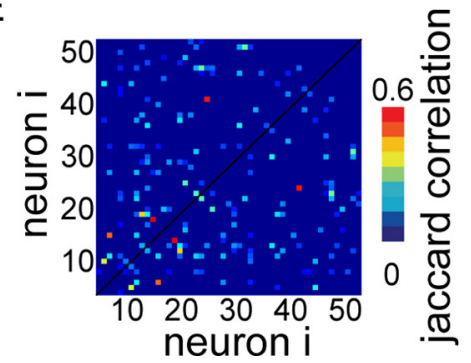

Figure 2. Network dynamics in control and denervated striatum. $A$, Raster plots of spontaneously active neurons in the normal striatum (naive rat; left) and in the DA-depleted striatum (right). Each row represents an active cell. Histograms below illustrate the percentage of coactive neurons along time. Peaks of spontaneous synchronous activity (red) indicate sets of neurons with simultaneous recurrent bursting. Significance level was set at $p<0.01$-dashed line. Patterns of synchronous activity are only present in the DA-depleted striatum. $\boldsymbol{B}$, Electrical activity recorded from a medium spiny neuron in the DA-depleted striatum. Notice spontaneous bursting (arrow) and spontaneous synaptic events (arrowhead). C, Spatial map of neurons exhibiting spontaneous calcium signals in the DA-depleted striatum (filled circles). Empty circles indicate cells inactive during the time of recording, but which responded to high $\mathrm{K}^{+}$depolarization with fluorescence increases. Red circles point to cells firing during peaks of synchronous activity. Calibration bar, $100 \mu \mathrm{m}$. D. Correlation map of spontaneously active cells. Cell pairs whose firing was significantly correlated at some moment are joined by a line (see Materials and Methods). $\boldsymbol{E}$, Cross-correlation matrix of all possible neuron pairs from $\boldsymbol{C}$ and $\boldsymbol{D}$. Note heterogeneous distribution of correlation coefficients.

at a given time (Figs. 3B, $4 B$ ). To choose the optimal number of states depicted from the LLE reduction, we used hard and fuzzy clustering algorithms taking the Dunn's index as a validity function (Sasaki et al., 2007; Carrillo-Reid et al., 2008, 2009a). To determine which neurons belong to each state, hierarchical cluster analysis was computed using Euclidean distances and the nearest neighbor single linkage method (Systat) (Figs. 3F, 4F).

Finally, number of active neurons as well as number of significant synchronous peaks, were compared in the same slices before and after drug applications (Wilcoxon $t$ test) or between control and DA-depleted slices or DA-depleted and DA-depleted slices plus the application of some drugs (Mann-Whitney $U$ test) for epochs lasting the same time.

\section{Results}

\section{Optical and electrophysiological recordings of fluo-4 loaded} striatal neurons

The simultaneous recording of electrical activity of a large number of cells with single cell resolution is required for the proper description of local network dynamics. Network or microcircuit dynamics is defined as the recurrent and alternating changes of correlated and synchronous activity generated by identified groups of neurons along time in a determined field of view. Correlated and synchronous firing is detected by the spontaneous firing together of several neurons, so-called synchrony peaks. We have previously shown that $\mathrm{Ca}^{2+}$ imaging allows record firing of dozens of striatal neurons as transient fluorescence changes (Fig. 1) (Carrillo-Reid et al., 2008). Image sequences from a field of view $800 \times 600 \mu \mathrm{m}$ in size of the dorsal striatum were recorded in 67 corticostriatal slices from both nondepleted (control) and DAdepleted sides (Fig. 1A), as well as from slices obtained from intact nonlesioned rats (naive) (see Materials and Methods). Electrophysiologically, recorded neurons show characteristics of medium spiny projection neurons (MSNs) such as inward recti- fication and a delayed firing of the first action potential (Fig. $1 B, C)$. A representative field of view containing fluo-4 loaded neurons is shown in Figure $1 D$. A semiautomatic contour detection algorithm defined the spatial distribution of all active and inactive neurons (Fig. $1 \mathrm{E}$, filled and empty contours, respectively) in each experiment (see Materials and Methods). The $\mathrm{Ca}^{2+}$ transients generated by all active cells were followed along time. Figure $1 F$ exemplifies representative $\mathrm{Ca}^{2+}$ transients from four spontaneously active cells. Evoked firing of two or more action potentials in MSNs elicits significant $\mathrm{Ca}^{2+}$ transients (Fig. $1 G$ ) ensuring that optical methods can accurately reflect electrical activity. In fact, the intensity of fluorescence change is proportional to the number of action potentials fired (Fig. $1 G, H$ ). Simultaneous $\mathrm{Ca}^{2+}$ imaging and electrophysiological recordings showed that the first derivative of $\mathrm{Ca}^{2+}$ transient represents the duration of the electrical activity that evoked it (Fig. $1 \mathrm{H}$ ) (Carrillo-Reid et al., 2008, 2009a). This allowed us to reconstruct the electrical activity of each recorded neuron in the network, represented as dots in a raster plot (Fig. $1 \mathrm{H}$; black dots at the bottom represent bursting activity).

\section{Striatal network dynamics in DA-depleted microcircuits}

Striatal neurons in control or naive slices remained mostly silent, with only a few neurons showing spontaneous activity, as seen in the raster plot (Fig. 2A, left) (Liang et al., 2008). These few active neurons lacked significant correlated activity (bottom; significance level was set at $p<0.01$; dashed line) (Crutcher and DeLong, 1984; Kimura, 1992; Carrillo-Reid et al., 2008). In contrast, in DA-depleted slices from 6-OHDA-treated rats, the number of neurons exhibiting spontaneous firing increased greatly (Fig. 2A; DA-depleted, notice the change in $y$-axis scale) (Galarraga et al., 1987; Raz et al., 1996; Tang et al., 2001; Tseng et al., 
2001; Goldberg et al., 2004; Costa et al., 2006; Liang et al., 2008). It has been hypothesized that hyperactivity in DAdepleted striatum is accompanied by increased synchronization of active neurons leading to an impaired selection of motor synergies (Uhlhaas and Singer, 2006; Hammond et al., 2007; Fuentes et al., 2009; Zold et al., 2009). We demonstrate this hypothesis showing that the number of neurons exhibiting correlated firing increases significantly in DA-depleted striatum as revealed by the appearance of numerous and significant synchrony peaks (Fig. 2A, right; DA-depleted; at the bottom synchrony peaks are shown in red with asterisks). Spontaneous synchrony peaks denote the enhanced correlated and synchronous activity of identified groups of neurons (Cossart et al., 2003; Ikegaya et al., 2004; Carrillo-Reid et al., 2008). The number of synchrony peaks per $180 \mathrm{~s}$ time epochs was (mean \pm SEM): $11.3 \pm 1.8$ in the DA-depleted side compared with $0.9 \pm 0.9$ in the control, non-DA-depleted side $(p<$ 0.0001; Mann-Whitney $U$ test, $n=17$ slices; both sides taken from the same rats). Moreover, spontaneous synchrony peaks were detected in all DA-depleted slices $(n=$ 54 slices) and very rarely in control or naive slices. Targeted whole-cell recordings from cells participating in the synchrony peaks showed spontaneous depolarization, bursting and frequent spontaneous synaptic events (Fig. $2 B$, arrow and arrowhead, respectively) (Galarraga et al., 1987; Tang et al., 2001; Zold et al., 2009). Spontaneous bursting induced by DA-depletion is rarely observed in control or naive striatal neurons and appeared variable, that is, it shows more kinds of bursting activity (Fig. $2 \mathrm{~B}$; note three kinds of burst) than bursting induced in naive slices by NMDA (Carrillo-Reid et al., 2009a). Enhanced, spontaneous, correlated and synchronous bursting denotes a remarkable change of network dynamics in the pathological tissue compared with the control or naive tissues.

The spatial distribution of identified neurons firing in synchrony showed groups of neurons close together as well as neurons that were hundreds of micrometers apart with numerous silent cells in between (Fig. 2C, filled circles; empty circles are inactive cells). Spatial correlation maps (Fig. 2D) showed that many of the active neurons exhibited a statistically significant correlated activity $(p<0.01)$. Lines connecting neurons indicate correlated firing at some moment during recording. Line thickness is proportional to the extent of correlation. On average, $96 \pm 3 \%$ of active cells at a given moment showed correlated firing $(n=20$ slices). Most correlated firing was produced by cells active during the synchrony peaks (Fig. 2D, filled red circles). Cross-correlation maps of network activity (Fig. $2 E ; p<0.01$ ) denoted that the degree of

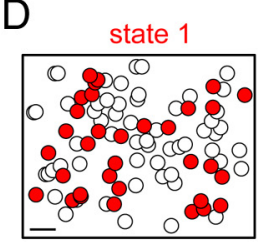
dominant state (blue)
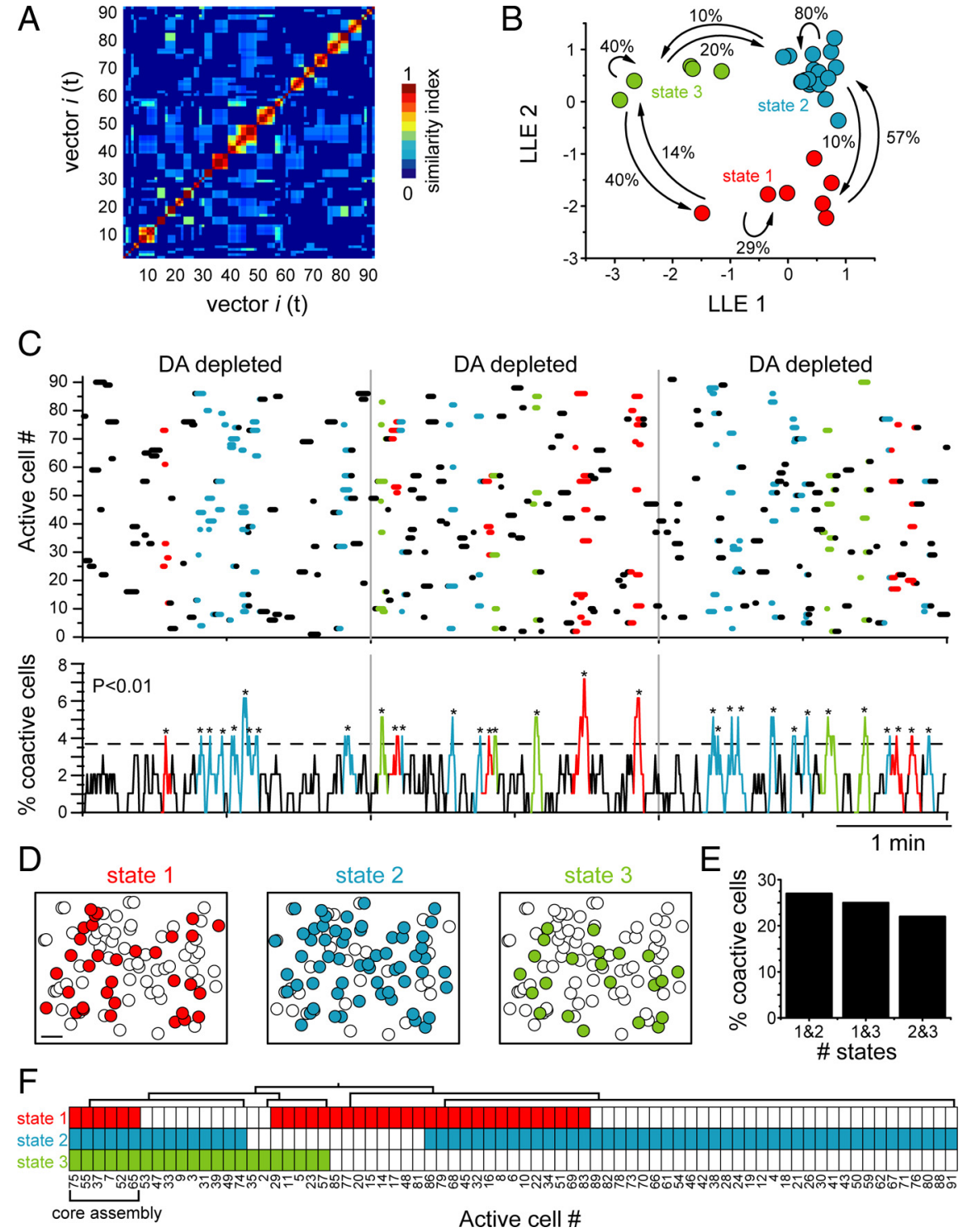

Figure 3. Engagement of striatal neuronal assembles within a dominant network state. $\boldsymbol{A}$, Matrix of similarity indexes of all vectors representing network dynamics as a function of time in DA-depleted striatum. $\boldsymbol{B}$, Multidimensional reduction using locally linear embedding (LLE). Each point represents a vector at a given time (see Materials and Methods). Different colors represent different network states. Percentages indicate network probability of leaving a given state. LLE reveals a dominant state (blue active during the time of recording (DA-depleted striatum). Colors denote cells pertaining to different states. Note increased recurrent activity of a dominant state (blue). D, Spatial distribution of the neuron pools supporting the different states. $\boldsymbol{E}$, Cells cluster analysis identifies numbered cells pertaining to each state (1,2 and 3). Note that only a few cells do not belong to the

correlation among active neurons was heterogeneous; involving diverse sets of cells bursting simultaneously.

As our data clearly show, spontaneous neuronal activity is considerably higher in DA-depleted striatum and this neuronal activity is characterized by spontaneous peaks of synchrony rarely seen in control slices (Cossart et al., 2003; Sasaki et al., 2007; Carrillo-Reid et al., 2008). However, in naive striatal slices sustained network dynamics can be induced by NMDA. This dynamics is characterized by spontaneous peaks of synchrony such that specific groups of neurons exhibit correlated firing in a recurrent way, alternating their activity with other groups of neurons, conforming self-organized spatiotemporal patterns that have been called cell assemblies (Hebb, 1949; Harris, 2005; 


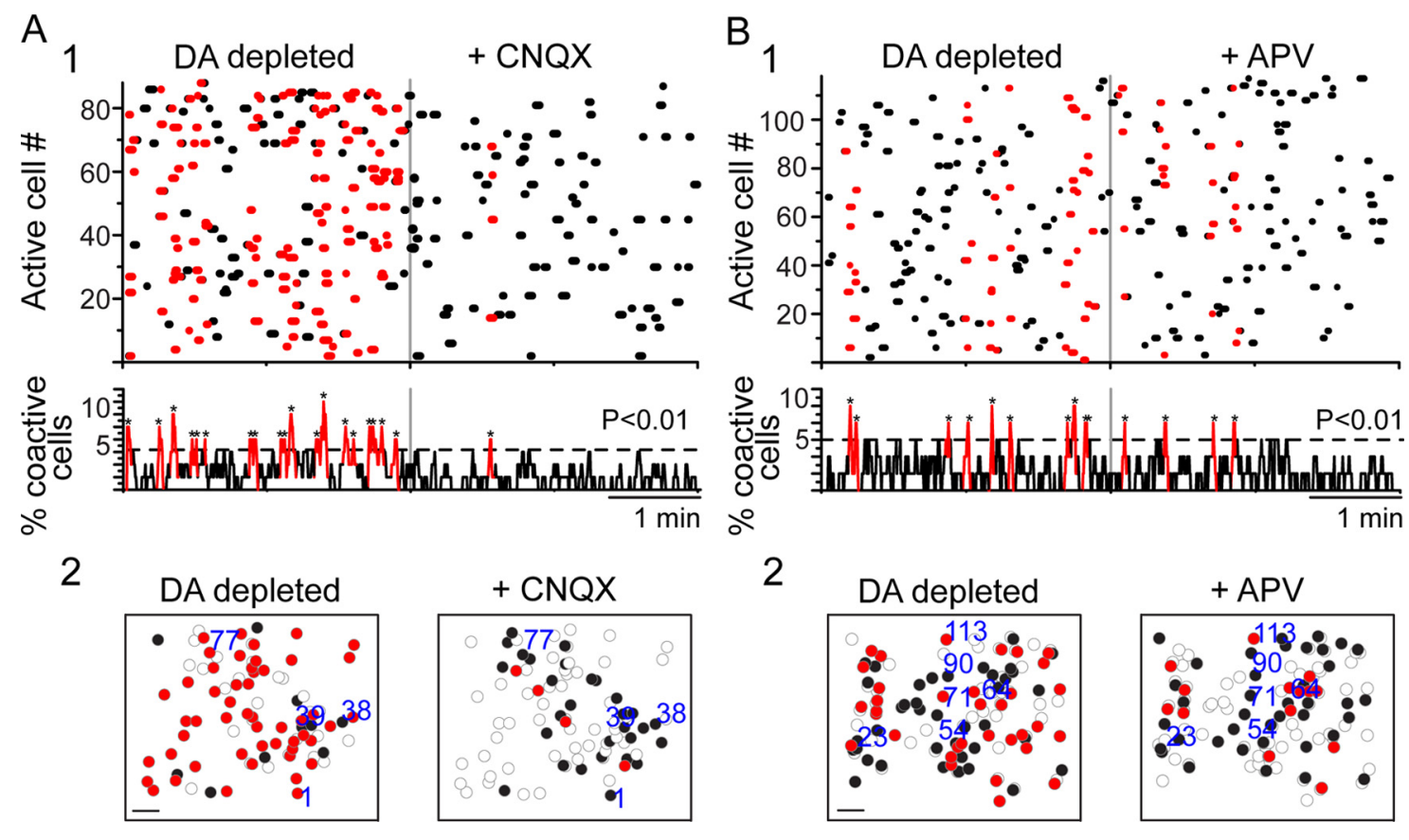

Figure 4. Inhibition of glutamatergic excitatory transmission on network dynamics of dopamine-depleted striatum. A1, Raster plot (top) and activity histogram (bottom) depicting network activity in the lesioned striatum of a 6-OHDA-treated rat before (left) and after the application of CNQX (right). Neuronal hyperactivity and peaks of synchrony (red, asterisks) were almost abolished after CNQX application. A2, Spatial maps showing the position of active neurons. Filled circles represent cells active during time of recording; red circles represent neurons involved in peaks of synchronous activity in control and CNQX. Blue numbers identify the position of some cells that were active in both conditions. B1, Raster plot (top) and activity histogram (bottom) illustrating network activity in the depleted striatum of a 6-OHDA-treated animal before (left) and after APV application (right). Peaks of synchronous activity (asterisks) were significantly reduced after APV application. B2, Spatial maps of cells active during the experiment (filled circles) and during the peaks of synchrony (red circles). Position of some cells active in both conditions are indicated with blue numbers.

Carrillo-Reid et al., 2008, 2009a). These cell assemblies can be modulated by transmitters (Vautrelle et al., 2009; Carrillo-Reid et al., 2009b). Therefore, we decided to compare the properties of the endogenously generated network activity of DA-depleted striatal microcircuits to the network activity induced by NMDA already reported in naive striatum (Carrillo-Reid et al., 2008).

\section{Network states and striatal cell assemblies in} dopamine-depleted slices

To study network states in the DA-depleted slices, the spontaneous peaks of synchronous activity were identified and a vector was formed from the data of each peak (bin $=250 \mathrm{~ms}$ ). Vectors depict the network behavior of the striatal microcircuit during periods of synchrony (see Materials and Methods). A network state is defined as a set of $\mathrm{N}$ vectors (bins) in $\mathrm{D}$ dimensions (active neurons) (Schreiber et al., 2003; Brown and Williams, 2005; Sasaki et al., 2006, 2007). Similarity indexes (see Materials and Methods) showed patterned cluster-like structures (mosaics) and similar behavior between different vectors (Fig. 3A).

To extract microcircuit dynamics from multidimensional data, the dimensionality of the network was reduced using Locally Linear Embedding (LLE; see Materials and Methods), a nonlinear dimensionality reduction technique (Roweis and Saul, 2000; Stopfer et al., 2003; Brown and Williams, 2005; CarrilloReid et al., 2008, 2009a). With this procedure, vectors can be projected in two dimensions (Fig. 3B). Dimensional reduction revealed that network dynamics in the DA-depleted striatum is characterized by a dominant state that comprised most peaks of synchrony (vectors) (Fig. 3B, blue circles; percentages indicate the network's probability of leaving a given state). Thus, the probability of a dominant state appearing in DA-depleted striatum is significantly higher (recurrence) than the probability of appearance of any other state (Fig. 3B). The neuron pools that generate network states in the DA-depleted slices were recorded with brief movies at given time intervals (epochs) then raster plots representing the activity of all neurons involved were built (Fig. 3C).

The neural activity of the DA-depleted striatum is characterized by numerous spontaneous peaks of synchronous activity per epoch (Fig. 3C, top, bottom). In addition to having a dominant state with a higher probability of appearance, DA-depleted striatal network comprises many cells that belong to different network states (Fig. 3D). The dominant state attracts most of these cells: $80 \pm 13 \%$ (Fig. $3 E ; n=17$ slices) as confirmed by hierarchical cluster analysis (Fig. $3 F$ ). Few active cells in the network are not associated to the dominant state. These experiments demonstrate that spontaneous network dynamics of the DA-depleted striatal microcircuit is remarkably different from that of non-DAdepleted contralateral (control) striatum, or striatal tissue from naive rats.

\section{Synaptic mechanisms underlying network states in DA-depleted slices}

DA depletion increases the glutamatergic synaptic activity in MSNs (Galarraga et al., 1987; Tang et al., 2001; Tseng et al., 2001; Bamford et al., 2004). We therefore sought to determine the role of glutamatergic and GABAergic synaptic transmission in the network dynamics of DA-depleted slices. Application of the AMPA/KA receptor antagonist CNQX $(10 \mu \mathrm{M})$, reduced the number of active cells from $82 \pm 12$ cells to $25 \pm 10$ cells per field $(p<0.006$; Wilcoxon $t$ test, $n=10$ slices), and significantly reduced the number of peaks of synchrony from $11 \pm 2$ to $1 \pm 0.9$ peaks per 180 s epoch ( $p<0.005$; Wilcoxon $t$ test, $n=10$ slices) (Fig. $4 A$ ). Similarly, upon inhibition of NMDA receptors with their an- 
tagonist APV $(50 \mu \mathrm{M})$, the number of active cells decreased from $83 \pm 14$ cells to $33 \pm 12$ cells $(p<0.006$; Wilcoxon $t$ test, $n=15$ slices) and the number of peaks of synchrony dropped from $11 \pm 2$ to $4 \pm 2$ peaks per $180 \mathrm{~s}$ epochs $(p<0.0007$; Wilcoxon $t$ test, $n=15$ slices) (Fig. $4 B$ ). Interestingly, some neurons remained active even in the presence of both glutamatergic antagonists.

Intrastriatal connectivity is largely mediated by GABAergic transmission (Czubayko and Plenz, 2002; Tunstall et al., 2002; Guzmán et al., 2003; Koos et al., 2004; Tepper et al., 2004; Mizuno et al., 2007; Tecuapetla et al., 2007, 2009) which includes GABAergic synapses between MSNs known to be important in sustaining bursting (Flores-Barrera et al., 2009). GABAergic transmission is also crucial to maintain a continuum of state transitions in the NMDA-treated striatal microcircuit (Carrillo-Reid et al., 2008). It has been shown that DA modulates the synaptic connections between MSNs (Guzmán et al., 2003; Tecuapetla et al., 2007, 2009), and that under conditions of DA-depletion, GABAergic transmission between striatal MSNs is reduced (Taverna et al., 2008; Tecuapetla et al., 2009).

We therefore studied striatal network changes after the blockade of fast GABAergic transmission with $10 \mu \mathrm{M}$ bicuculline (Fig. 5). Since DA-depletion reduces inhibitory connectivity among striatal MSNs (Taverna et al., 2008; Tecuapetla et al., 2009) it was predicted that the actions of GABAergic transmission blockade on the overall activity of the network were partially occluded. We found that GABAergic blockade is not reflected in the number of peaks per $180 \mathrm{~s}$ epoch: $12 \pm 2$ before vs $10 \pm 7$ after bicuculline $(n=18 ; p>0.36$, Wilcoxon $t$ test), confirming the prediction. However, similarity indexes maps showed an increase in the patterned cluster-like structures before and after the application of bicuculline (Fig. $5 A$ ). In fact, after the blockade of GABAergic transmission the sharing (overlapping) of neurons between different vectors changed from $24 \pm 3 \%(n=25)$ in DA-depleted circuits vs $32 \pm 3 \%(n=23)$ after bicuculline was added to these circuits ( $p<0.001$; Mann-Whitney $U$ test; $n=18$ slices). This is reflected as a restriction in the diversity of network states (Fig. $5 B$ ). The loss of GABAergic transmission further facilitated the entrainment of neuronal vectors into a dominant state (Fig. $5 B$ ). The dominant state traps most active neurons (Fig. $5 C$; see recurrent yellow synchrony peaks). Figure $5 D$ shows the spatial distribution of neuron pools involved in each network state; note number of neurons participating in the state denoted by yellow synchrony peaks. Cells shared by different states in both conditions are shown in Figure $5 E$. bicuculline (bottom).
B
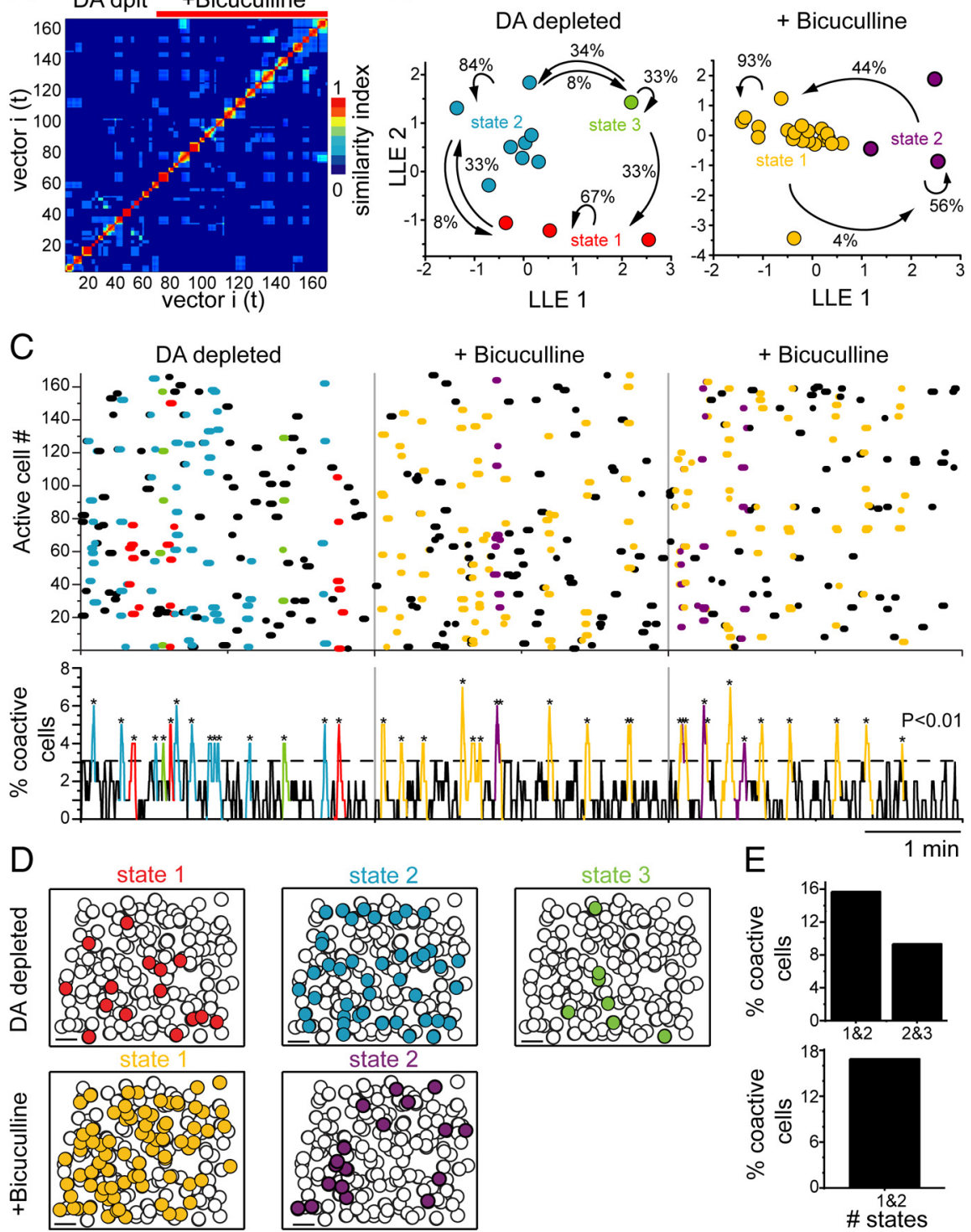

Figure 5. Suppression of fast GABAergic inhibition on network dynamics in the dopamine-depleted striatum. $\boldsymbol{A}$, Similarity index matrix of the denervated striatum in the DA-depleted condition (DA dplt) and after the application of the $G_{A B A}$ receptor blocker bicuculline (10 $\mu \mathrm{m}$, red line). $\boldsymbol{B}$, LLE projection showing cell assemblies of the denervated striatum before (left) and after the application of bicuculline (right). Note that after the application of bicuculline the number of network states is reduced. $\boldsymbol{C}$, Raster plot (top) and time histogram of the overall activity (bottom) depicting network dynamics in the denervated striatum before (left) and after bicuculline application (middle and right). Colors denote cells belonging to the different network states before and after the application of bicuculline. $\boldsymbol{D}$, Spatial distribution of neuronal pools pertaining to the different network states before (top) and after the application of bicuculline. $\boldsymbol{E}$, Percentage of coactive cells in the DA-depleted condition (top) and in the presence of

In an attempt to rescue the microcircuit from the dominant state appearing after DA-depletion, we then performed acute applications of a dopamine receptor agonist. The similarity map of a representative experiment showed a change in the patterned structures after the application of a $1 \mu \mathrm{M}$ concentration of the $\mathrm{D}_{1}$-class receptor agonist, SKF 81297 (Fig. 6A). A brief image sequence was enough to corroborate the presence of a dominant network state during DA-depletion (Fig. 6B, left). Exposure to SKF 81297, decreased the number of shared neurons between different vectors, from $26 \pm 3 \%(n=20)$ in DA-depleted circuit vs $20 \pm 3 \%(n=29)$ after adding the $\mathrm{D}_{1}$-receptor agonist $(p<$ 0.001; Mann-Whitney $U$ test; $n=4$ slices). A decrease in the number of shared neurons is reflected as a partial restoration of 
A

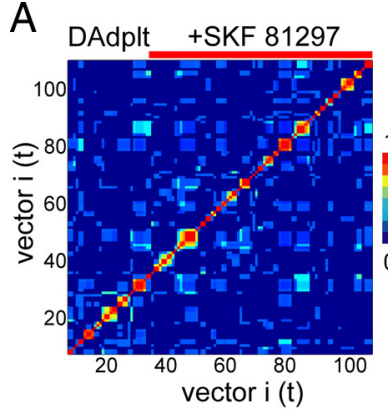

B
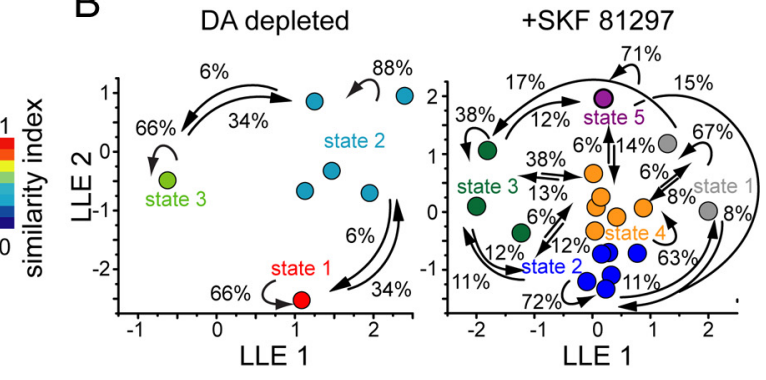

C DA depleted
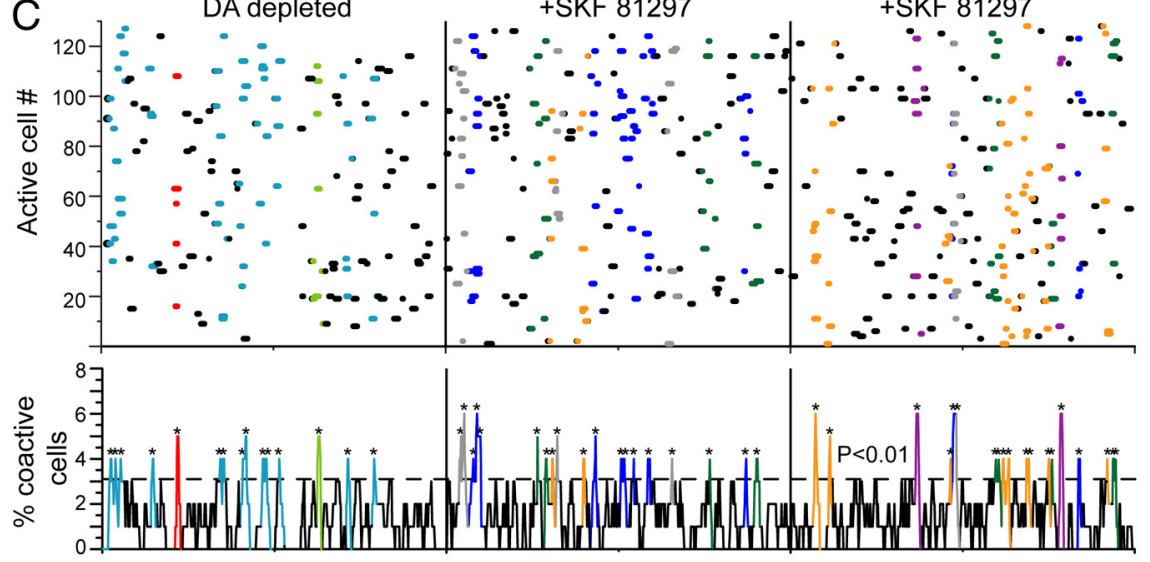

$\mathrm{D}$
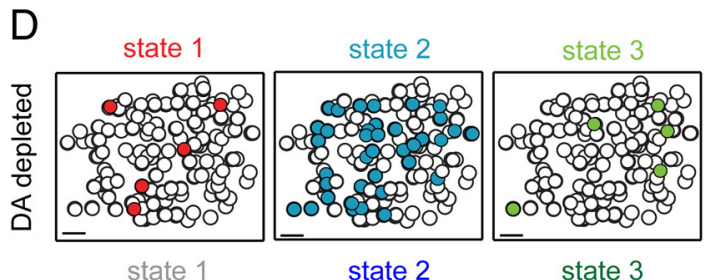

E

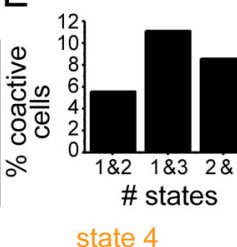

$1 \mathrm{~min}$
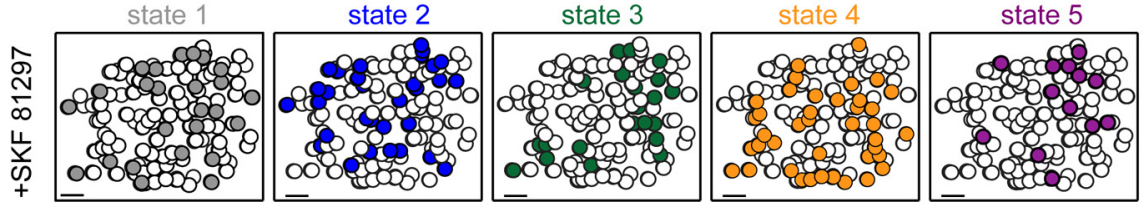

Figure 6. Effects of a dopamine receptor agonist. $A$, Similarity index matrix of the DA-depleted striatum before and after the application of a $D_{1}$ receptor agonist: SKF 81297, $1 \mu \mathrm{M}$ (red line). B, LLE projection showing cell assemblies of the DA-depleted striatum before (left) and after the application of SKF 81297 (right). Notice the appearance of different network states with no preference for a particular one (absence of a dominant state) after agonist application. C, Raster plot (top) and time histogram of the overall activity (bottom) depicting network dynamics in the DA-depleted striatum before (left) and after SKF 81297 application (middle and right). Colors denote cells belonging to the different network states before and after the application of SKF 81297. D, Spatial distribution of neuron pools pertaining to the different network states before (top) and after the application of SKF 81297 (bottom). $\boldsymbol{E}$, Percentage of coactive cells in the DA-depleted condition before (left) and in the presence of SKF 81297 (right).

\section{Discussion}

This study demonstrates that spontaneous network dynamics of DA-depleted striatal microcircuits is fundamentally different from that observed in striatal slices from either control non-DA-depleted, or naive rats. Striatal neurons, which are mostly silent under control conditions, become hyperactive and often synchronized in the denervated striatum, with a dominant network state that engages most active neurons. This behavior also differs from NMDA-induced network dynamics in the normal striatum, where network states are defined by specific groups of neurons firing in synchrony with recurrent and alternate activity. Network hyperactivity and entrainment of active neurons into a dominant state is antagonized or enhanced by glutamate receptor or $\mathrm{GABA}_{\mathrm{A}}$ receptor antagonists, respectively. Network entrainment into a dominant state ceased after the addition of a dopamine receptor agonist. To observe all these behaviors in a small field of view suggests that unit microcircuits can be seen as modules whose similarity and coordination results in the function of more extended circuits, constituting the interface between single-cell neuronal activity and global brain function. Their understanding may unveil the intrinsic and synaptic rules that govern normal and pathological circuit behaviors: Our results support the notion that DA-depletion is correlated with increased, not decreased activity of striatal microcircuits. These results are also consistent with a decreased inhibition between MSNs combined with an increased excitatory drive in the DAdepleted striatum.

\section{Network dynamics in DA-depleted striatal local networks}

Striatal neurons in vivo exhibit infrequent spontaneous activity during physiological states unrelated to task performance (Crutcher and DeLong, 1984; Kimura, 1992; Liang et al., 2008; Vautrelle et al.,

the network capability to generate different states (Fig. $6 \mathrm{~B}$, right). Contrary to the expected decrease of the overall activity of the network; after the application of a dopamine receptor agonist the striatal network remained hyperactive. Peaks of synchronous activity continued appearing (Fig. 6C), perhaps due to hypercholinergia (Carrillo-Reid et al., 2009b). The spatial distribution of the neurons belonging to different network states is shown in Figure $6 D$. Figure $6 E$ shows the cells shared by different states in DA-depleted condition and in the presence of SKF 81297.

The increase in the diversity of network states and activity traveling among them (Carrillo-Reid et al., 2008) is a good outcome when several agonists have still to be tested. A detailed exploration of the effects of diverse dopamine receptor agonists and antagonists in normal and pathological conditions deserves a study of its own (now underway).
2009). Loss of dopaminergic innervation leads to increased spontaneous activity in MSNs both in vitro and in vivo (Galarraga et al., 1987; Raz et al., 1996; Tseng et al., 2001; Goldberg et al., 2004; Day et al., 2006; Uhlhaas and Singer, 2006; Liang et al., 2008; Zold et al., 2009). Such hyperactivity has been associated with signs of Parkinson disease such as akinesia, rigidity and tremor (Costa et al., 2006; Liang et al., 2008; Fuentes et al., 2009; Walters and Bergstrom, 2009). Nonetheless, network mechanisms underlying hyperactivity have not been examined given the technical limitations of single cell recordings (Kimura, 1992; Courtemanche et al., 2003; Berke et al., 2004; Goldberg et al., 2004; Costa et al., 2006; Liang et al., 2008; Blume and Tseng, 2009; Fuentes et al., 2009). Here we demonstrate that striatal microcircuits from both control and 6-OHDA lesioned animals are accessible to dy- 
namic calcium imaging in corticostriatal slice preparations in vitro. Single cell resolution and recording from dozens of cells simultaneously with application of neurotransmitters and drugs through the bathing media uncover changes in network dynamics seen through vectorization and dimensional reduction (Cossart et al., 2003; Carrillo-Reid et al., 2008). These methods allow a rigorous comparison of different network states along time (Barnes et al., 2005; Sasaki et al., 2007).

Corticostriatal slices from control, untreated rats, exhibit seldom activity. Moreover, it has been shown that network dynamics induced by NMDA in control striatum involves several network states with similar probability of appearance. Neuronal pools underlying these states alternate their synchronized activity depicting spatiotemporal patterns of self-organized recurrent pathways (Carrillo-Reid et al., 2008, 2009a). The flow of neural activity between states following quasi-fixed trajectories has been considered a property of cell assemblies (Hebb, 1949; Harris, 2005). By contrast, the DA-depleted circuit is characterized by the emergence of a dominant state with a high probability of recurrence and absorbing most active neurons in the field of view. State transitions decrease due to this highly recurrent state which impairs alternation between neuron pools, perhaps correlating with impaired switching between motor acts or programs (Olsson et al., 1995; Hutchison et al., 2004; Uhlhaas and Singer, 2006; Fuentes et al., 2009; Walters and Bergstrom, 2009). But despite having a reduced number of states, spontaneous peaks of synchronization increase in frequency, neural activity being significantly higher than that evoked with NMDA. Recovery of sequences of activity may need the eradication of the dominant state to restore activity cycles (Grillner, 2006). Here we attained a partial recovery by addition of a dopamine agonist.

\section{Mechanisms underlying anomalous network behavior in the DA-depleted striatum}

Parkinson disease signs emerge after the loss of striatal dopaminergic innervation (Carlsson, 1972; Zgaljardic et al., 2003). Dopamine depletion enhances the corticostriatal excitatory drive being this a main source of hyperactivity (Galarraga et al., 1987; Tang et al., 2001; Tseng et al., 2001; Liang et al., 2008; Blume and Tseng, 2009; Zold et al., 2009). In addition, decreased inhibition among principal cells (Taverna et al., 2008; Tecuapetla et al., 2009) has been demonstrated, becoming a second source of hyperexcitability (Costa et al., 2006; Carrillo-Reid et al., 2008). Accordingly, we show that blockade of glutamatergic synapses reduced both hyperactivity and synchrony in DA-depleted circuits. Activation of striatal neurons through AMPA receptors resulted crucial for pathological network dynamics because their blockade produced a dramatic decrease in the number of synchrony peaks (Day et al., 2006; Calabresi et al., 2007; DeLong and Wichmann, 2007; Flores-Barrera et al., 2009). NMDA receptors inhibition also decreased hyperactivity and synchrony, indicating that plateau potentials tune the output of striatal networks (Schiller and Schiller, 2001; Vergara et al., 2003; Carrillo-Reid et al., 2008; Flores-Barrera et al., 2009). Enhanced excitatory drive (Galarraga et al., 1987; Tseng et al., 2001; Bamford et al., 2004; Calabresi et al., 2007) is perhaps caused by both lack of presynaptic modulation of glutamatergic afferents mediated by dopamine (Flores-Hernández et al., 1997; Tang et al., 2001; Bamford et al., 2004) and secondary alterations in other transmitter systems and neuronal intrinsic properties (Galarraga et al., 1999; Day et al., 2006; Carrillo-Reid et al., 2009b).

Inhibitory connections between MSNs are essential for the alternation of activity among different neuronal pools as well as for sustaining plateau potentials and bursting (Carrillo-Reid et al., 2008; Flores-Barrera et al., 2009). When GABAergic synapses are blocked in the NMDA-treated normal striatum, increased network activity becomes engaged into a dominant network state (Carrillo-Reid et al., 2008). Thus, entrainment into a dominant state may depend on a limited interaction among striatal neurons (Mizuno et al., 2007; Tecuapetla et al., 2007, 2009; Taverna et al., 2008). However, DA-blockade does not disrupt strong connections among striatopallidal $\mathrm{D}_{2}$-receptor-containing neurons (Tecuapetla et al., 2009) and inhibitory synapses from GABAergic interneurons (Tepper et al., 2004; Tecuapetla et al., 2007; Dehorter et al., 2009), suggesting that specific GABAergic synapses have different roles in modulating network dynamics (Berke et al., 2004; Carrillo-Reid et al., 2008; Gittis et al., 2010; Planert et al., 2010). Blockade of remaining inhibition in the DA-depleted striatum produces a more pronounced dominant state, suggesting that intrastriatal connectivity is essential in circuitry processing for the transformation of cortical commands into spatiotemporal patterned output.

\section{Physiological relevance}

Synchronous neuronal firing reflects coordinated activity of specific networks (Singer, 1999; Barnes et al., 2005; Fries et al., 2007; Carrillo-Reid et al., 2008; Graybiel, 2008). Sequential synchronous activation of neuronal pools generates ordered spatiotemporal patterns that may explain procedural memory processes and habit formation (Barnes et al., 2005; Harris, 2005; Graybiel, 2008; Uhlhaas et al., 2009). Pathological states result from rules similar to those governing physiological states, but in a more frequent and less modulated situation (Costa et al., 2006; Carrillo-Reid et al., 2008, 2009a; Walters and Bergstrom, 2009). Thus, epilepsy, schizophrenia, compulsive disorder, dementia, Alzheimer and Parkinson disease have been associated with abnormal processes of synchronization (Uhlhaas and Singer, 2006; Hammond et al., 2007; Kreitzer and Malenka, 2008; Uhlhaas et al., 2009) whose cellular mechanisms are still not understood.

We here demonstrate that a dramatic reconfiguration of striatal network dynamics occurs at the microcircuit level after DAdepletion. Bizarre hyperactivity and enhanced synchronization may induce anomalous pattern generators (Yin et al., 2009; Carrillo-Reid et al., 2009a) comprised by dominant neuronal pools that absorb cell assemblies forcing them to execute repeatedly the same configuration thus impeding the normal flow of activity. In the long term (chronicity), these anomalies may lead to robust modifications of microcircuit connectivity through plastic mechanisms (Pisani et al., 2005; Day et al., 2006). When a given proportion of cell assemblies get tied up into these anomalous unproductive states, the availability of neuronal pools required for the execution of voluntary procedures may be permanently decreased (Brown, 2007; Hammond et al., 2007; Fuentes et al., 2009). The present experiments show that calcium imaging techniques in the slice preparation of denervated striatum can help to distinguish between normal and abnormal microcircuit behaviors, and also provide clues to understand the physiological actions of potential therapeutic agents, thus paving the way for a novel in vitro bioassay characterization.

\section{References}

Bamford NS, Zhang H, Schmitz Y, Wu NP, Cepeda C, Levine MS, Schmauss C, Zakharenko SS, Zablow L, Sulzer D (2004) Heterosynaptic dopamine neurotransmission selects sets of corticostriatal terminals. Neuron 42:653-663.

Barnes TD, Kubota Y, Hu D, Jin DZ, Graybiel AM (2005) Activity of striatal 
neurons reflects dynamic encoding and recoding of procedural memories. Nature 437:1158-1161.

Berke JD, Okatan M, Skurski J, Eichenbaum HB (2004) Oscillatory entrainment of striatal neurons in freely moving rats. Neuron 43:883-896.

Blume SR, Tseng KY (2009) Leading toward a unified cortico/basal ganglia functional model. In: Cortico-subcortical dynamics in Parkinson disease (Tseng KY, ed), pp 3-22. New York: Humana/Springer.

Brown P (2007) Abnormal oscillatory synchronisation in the motor system leads to impaired movement. Curr Opin Neurobiol 17:656-664.

Brown P, Williams D (2005) Basal ganglia local field potential activity: character and functional significance in the human. Clin Neurophysiol 116:2510-2519.

Calabresi P, Picconi B, Tozzi A, Di Filippo M (2007) Dopamine-mediated regulation of corticostriatal synaptic plasticity. Trends Neurosci 30:211-219.

Carlsson A (1972) Biochemical and pharmacological aspects of Parkinsonism. Acta Neurol Scand Suppl 51:11-42.

Carrillo-Reid L, Tecuapetla F, Tapia D, Hernández-Cruz A, Galarraga E, Drucker-Colin R, Bargas J (2008) Encoding network states by striatal cell assemblies. J Neurophysiol 99:1435-1450.

Carrillo-Reid L, Tecuapetla F, Ibáñez-Sandoval O, Hernández-Cruz A, Galarraga E, Bargas J (2009a) Activation of the cholinergic system endows compositional properties to striatal cell assemblies. J Neurophysiol 101:737-749.

Carrillo-Reid L, Tecuapetla F, Vautrelle N, Hernández A, Vergara R, Galarraga E, Bargas J (2009b) Muscarinic enhancement of persistent sodium current synchronizes striatal medium spiny neurons. J Neurophysiol 102:682-690.

Cossart R, Aronov D, Yuste R (2003) Attractor dynamics of network UP states in the neocortex. Nature 423:283-288.

Costa RM, Lin SC, Sotnikova TD, Cyr M, Gainetdinov RR, Caron MG, Nicolelis MA (2006) Rapid alterations in corticostriatal ensemble coordination during acute dopamine-dependent motor dysfunction. Neuron 52:359-369.

Courtemanche R, Fujii N, Graybiel AM (2003) Synchronous, focally modulated beta-band oscillations characterize local field potential activity in the striatum of awake behaving monkeys. J Neurosci 23:11741-11752.

Crutcher MD, DeLong MR (1984) Single cell studies of the primate putamen. II. Relations to direction of movement and pattern of muscular activity. Exp Brain Res 53:244-258.

Czubayko U, Plenz D (2002) Fast synaptic transmission between striatal spiny projection neurons. Proc Natl Acad Sci U S A 99:15764-15769.

Day M, Wang Z, Ding J, An X, Ingham CA, Shering AF, Wokosin D, Ilijic E, Sun Z, Sampson AR, Mugnaini E, Deutch AY, Sesack SR, Arbuthnott GW, Surmeier DJ (2006) Selective elimination of glutamatergic synapses on striatopallidal neurons in Parkinson disease models. Nat Neurosci 9:251-259.

Dehorter N, Guigoni C, Lopez C, Hirsch J, Eusebio A, Ben-Ari Y, Hammond C (2009) Dopamine-deprived striatal GABAergic interneurons burst and generate repetitive gigantic IPSCs in medium spiny neurons. J Neurosci 29:7776-7787.

DeLong MR, Wichmann T (2007) Circuits and circuit disorders of the basal ganglia. Arch Neurol 64:20-24.

Dunnett SB, Björklund A, Stenevi U, Iversen SD (1981) Behavioural recovery following transplantation of substantia nigra in rats subjected to 6-OHDA lesions of the nigrostriatal pathway. I. Unilateral lesions. Brain Res 215:147-161.

Flores-Barrera E, Laville A, Plata V, Tapia D, Bargas J, Galarraga E (2009) Inhibitory contribution to suprathreshold corticostriatal responses: an experimental and modeling study. Cell Mol Neurobiol 29:719-731.

Flores-Hernández J, Galarraga E, Bargas J (1997) Dopamine selects glutamatergic inputs to neostriatal neurons. Synapse 25:185-195.

Fries P, Nikolić D, Singer W (2007) The gamma cycle. Trends Neurosci 30:309-316.

Fuentes R, Petersson P, Siesser WB, Caron MG, Nicolelis MA (2009) Spinal cord stimulation restores locomotion in animal models of Parkinson's disease. Science 323:1578-1582.

Galarraga E, Bargas J, Martínez-Fong D, Aceves J (1987) Spontaneous synaptic potentials in dopamine-denervated neostriatal neurons. Neurosci Lett 81:351-355.

Galarraga E, Hernández-López S, Reyes A, Miranda I, Bermudez-Rattoni F, Vilchis C, Bargas J (1999) Cholinergic modulation of neostriatal output: a functional antagonism between different types of muscarinic receptors. J Neurosci 19:3629-3638.

Gittis AH, Nelson AB, Thwin MT, Palop JJ, Kreitzer AC (2010) Distinct roles of GABAergic interneurons in the regulation of striatal output pathways. J Neurosci 30:2223-2234.

Goldberg JA, Rokni U, Boraud T, Vaadia E, Bergman H (2004) Spike synchronization in the cortex/basal-ganglia networks of Parkinsonian primates reflects global dynamics of the local field potentials. J Neurosci 24:6003-6010.

Grant RJ, Clarke PB (2002) Susceptibility of ascending dopamine projections to 6-hydroxydopamine in rats: effect of hypothermia. Neuroscience 115:1281-1294

Graybiel AM (2008) Habits, rituals, and the evaluative brain. Annu Rev Neurosci 31:359-387.

Grillner S (2006) Biological pattern generation: the cellular and computational logic of networks in motion. Neuron 52:751-766.

Guzmán JN, Hernández A, Galarraga E, Tapia D, Laville A, Vergara R, Aceves J, Bargas J (2003) Dopaminergic modulation of axon collaterals interconnecting spiny neurons of the rat striatum. J Neurosci 23:8931-8940.

Hammond C, Bergman H, Brown P (2007) Pathological synchronization in Parkinson's disease: networks, models and treatments. Trends Neurosci 30:357-364.

Harris KD (2005) Neural signatures of cell assembly organization. Nat Rev Neurosci 6:399-407.

Hebb DO (1949) The organization of behavior. New York: Wiley.

Hutchison WD, Dostrovsky JO, Walters JR, Courtemanche R, Boraud T, Goldberg J, Brown P (2004) Neuronal oscillations in the basal ganglia and movement disorders: evidence from whole animal and human recordings. J Neurosci 24:9240-9243.

Ikegaya Y, Aaron G, Cossart R, Aronov D, Lampl I, Ferster D, Yuste R (2004) Synfire chains and cortical songs: temporal modules of cortical activity. Science 304:559-564

Ingham CA, Hood SH, Taggart P, Arbuthnott GW (1998) Plasticity of synapses in the rat neostriatum after unilateral lesion of the nigrostriatal dopaminergic pathway. J Neurosci 18:4732-4743.

Kawaguchi Y, Wilson CJ, Emson PC (1989) Intracellular recording of identified neostriatal patch and matrix spiny cells in a slice preparation preserving cortical inputs. J Neurophysiol 62:1052-1068.

Kimura M (1992) Behavioral modulation of sensory responses of primate putamen neurons. Brain Res 578:204-214.

Koos T, Tepper JM, Wilson CJ (2004) Comparison of IPSCs evoked by spiny and fast-spiking neurons in the neostriatum. J Neurosci 24:7916-7922.

Kreitzer AC, Malenka RC (2008) Striatal plasticity and basal ganglia circuit function. Neuron 60:543-554.

Liang L, DeLong MR, Papa SM (2008) Inversion of dopamine responses in striatal medium spiny neurons and involuntary movements. J Neurosci 28:7537-7547.

Magill PJ, Bolam JP, Bevan MD (2001) Dopamine regulates the impact of the cerebral cortex on the subthalamic nucleus-globus pallidus network Neuroscience 106:313-330.

Mao BQ, Hamzei-Sichani F, Aronov D, Froemke RC, Yuste R (2001) Dynamics of spontaneous activity in neocortical slices. Neuron 32:883-898.

Mizuno T, Schmauss C, Rayport S (2007) Distinct roles of presynaptic dopamine receptors in the differential modulation of the intrinsic synapses of medium-spiny neurons in the nucleus accumbens. BMC Neurosci 8:8

Ni ZG, Bouali-Benazzouz R, Gao DM, Benabid AL, Benazzouz A (2001) Time-course of changes in firing rates and firing patterns of subthalamic nucleus neuronal activity after 6-OHDA-induced dopamine depletion in rats. Brain Res 899:142-147.

Olsson M, Nikkhah G, Bentlage C, Björklund A (1995) Forelimb akinesia in the rat Parkinson model: differential effects of dopamine agonists and nigral transplants as assessed by a new stepping test. J Neurosci 15:3863-3875.

Pisani A, Centonze D, Bernardi G, Calabresi P (2005) Striatal synaptic plasticity: implications for motor learning and Parkinson's disease. Mov Disord 20:395-402.

Planert H, Szydlowski SN, Hjorth JJ, Grillner S, Silberberg G (2010) Dynamics of synaptic transmission between fast-spiking interneurons and striatal projection neurons of the direct and indirect pathways. J Neurosci 30:3499-3507.

Raz A, Feingold A, Zelanskaya V, Vaadia E, Bergman H (1996) Neuronal 
synchronization of tonically active neurons in the striatum of normal and parkinsonian primates. J Neurophysiol 76:2083-2088.

Roweis ST, Saul LK (2000) Nonlinear dimensionality reduction by locally linear embedding. Science 290:2323-2326.

Sasaki T, Kimura R, Tsukamoto M, Matsuki N, Ikegaya Y (2006) Integrative spike dynamics of rat CA1 neurons: a multineuronal imaging study. J Physiol 574:195-208.

Sasaki T, Matsuki N, Ikegaya Y (2007) Metastability of active CA3 networks. J Neurosci 27:517-528.

Schiller J, Schiller Y (2001) NMDA receptor-mediated dendritic spikes and coincident signal amplification. Curr Opin Neurobiol 11:343-348.

Schreiber S, Fellous JM, Whitmer D, Tiesinga P, Sejnowski TJ (2003) A new correlation-based measure of spike timing reliability. Neurocomputing 52-54:925-935.

Schwartz TH, Rabinowitz D, Unni V, Kumar VS, Smetters DK, Tsiola A, Yuste R (1998) Networks of coactive neurons in developing layer 1. Neuron 20:541-552.

Singer W (1999) Neuronal synchrony: a versatile code for the definition of relations? Neuron 24:49-65, 111-125.

Stopfer M, Jayaraman V, Laurent G (2003) Intensity versus identity coding in an olfactory system. Neuron 39:991-1004.

Tang K, Low MJ, Grandy DK, Lovinger DM (2001) Dopamine-dependent synaptic plasticity in striatum during in vivo development. Proc Natl Acad Sci U S A 98:1255-1260.

Taverna S, Ilijic E, Surmeier DJ (2008) Recurrent collateral connections of striatal medium spiny neurons are disrupted in models of Parkinson's disease. J Neurosci 28:5504-5512.

Tecuapetla F, Carrillo-Reid L, Bargas J, Galarraga E (2007) Dopaminergic modulation of short-term synaptic plasticity at striatal inhibitory synapses. Proc Natl Acad Sci U S A 104:10258-10263.

Tecuapetla F, Koós T, Tepper JM, Kabbani N, Yeckel MF (2009) Differential dopaminergic modulation of neostriatal synaptic connections of striatopallidal axon collaterals. J Neurosci 29:8977-8990.

Tepper JM, Koós T, Wilson CJ (2004) GABAergic microcircuits in the neostriatum. Trends Neurosci 27:662-669.

Tseng KY, Kasanetz F, Kargieman L, Riquelme LA, Murer MG (2001) Cortical slow oscillatory activity is reflected in the membrane potential and spike trains of striatal neurons in rats with chronic nigrostriatal lesions. J Neurosci 21:6430-6439.

Tunstall MJ, Oorschot DE, Kean A, Wickens JR (2002) Inhibitory interactions between spiny projection neurons in the rat striatum. J Neurophysiol 88:1263-1269.

Uhlhaas PJ, Singer W (2006) Neural synchrony in brain disorders: relevance for cognitive dysfunctions and pathophysiology. Neuron 52:155-168.

Uhlhaas PJ, Pipa G, Lima B, Melloni L, Neuenschwander S, Nikolić D, Singer W (2009) Neural synchrony in cortical networks: history, concept and current status. Front Integr Neurosci 3:17.

Vautrelle N, Carrillo-Reid, L., Bargas, J. (2009) Diversity of up-state voltage transitions during different network states. In: Cortico-subcortical dynamics in Parkinson disease (Tseng KY, ed), pp 73-85. New York: Humana/Springer.

Vergara R, Rick C, Hernández-López S, Laville JA, Guzman JN, Galarraga E, Surmeier DJ, Bargas J (2003) Spontaneous voltage oscillations in striatal projection neurons in a rat corticostriatal slice. J Physiol 553:169-182.

Walters JR, Bergstrom DA (2009) Basal ganglia network synchronization in animal models of Parkinson's disease. In: Cortico-subcortical dynamics in Parkinson disease (Tseng KY, ed), pp 117-142. New York: Humana/Springer.

Wilson CL, Cash D, Galley K, Chapman H, Lacey MG, Stanford IM (2006) Subthalamic nucleus neurones in slices from 1-methyl-4-phenyl-1,2,3,6tetrahydropyridine-lesioned mice show irregular, dopamine-reversible firing pattern changes, but without synchronous activity. Neuroscience 143:565-572.

Yin HH, Mulcare SP, Hilário MR, Clouse E, Holloway T, Davis MI, Hansson AC, Lovinger DM, Costa RM (2009) Dynamic reorganization of striatal circuits during the acquisition and consolidation of a skill. Nat Neurosci 12:333-341.

Zgaljardic DJ, Borod JC, Foldi NS, Mattis P (2003) A review of the cognitive and behavioral sequelae of Parkinson's disease: relationship to frontostriatal circuitry. Cogn Behav Neurol 16:193-210.

Zold CL, Belluscio M, Kasanetz F, Pomata PE, Riquelme LA, Gonon F, Murer MG (2009) Converging into a unified model of Parkinson's disease pathophysiology. In: Cortico-subcortical dynamics in Parkinson disease (Tseng KY, ed), pp 143-156. New York: Humana/Springer. 